\title{
Z-Guggulsterone attenuates cognitive deficits and reduces amyloid- $\beta$ levels in APPswe/PS1dE9 mice by inhibiting toll-like receptor 4 pathway
}

Jing Liu

Chinese PLA General Hospital

\section{Yang Yang}

Chinese PLA General Hospital

\section{Yane Guo}

Chinese PLA General Hospital

\section{Yanchang Shang}

Chinese PLA General Hospital

\section{Bo Zhou}

Chinese PLA General Hospital

Jiao Fan

Chinese PLA General Hospital

Chao Wei ( $\nabla$ weichao301@163.com )

Chinese PLA General Hospital https://orcid.org/0000-0002-2982-9010

\section{Research}

Keywords: Z-Guggulsterone, Alzheimer's disease, TLR4, Inflammation, Amyloid- $\beta$ peptide

Posted Date: August 9th, 2021

DOl: https://doi.org/10.21203/rs.3.rs-763008/v1

License: (c) (i) This work is licensed under a Creative Commons Attribution 4.0 International License. Read Full License 


\section{Abstract \\ Background}

Accumulating evidence has indicated that inflammatory damage plays a vital role in the pathogenesis of Alzheimer's disease (AD) and represents one of the therapeutic targets. Z-Guggulsterone (Z-GS) is an active component extracted from myrrh, which has been widely used as an anti-inflammatory agent. The present study was to investigate the anti-inflammatory and neuroprotective effects of Z-GS on the disease progression, as well as the underlying mechanisms in the transgenic mouse models of AD.

\section{Methods}

Using behavioral, histological, and biochemical methods, outcomes of Z-GS treatment on neuropathology and cognitive deficits were studied in the APPswe/PS1dE9 mice.

\section{Results}

We found that Z-GS treatment resulted in a dramatic improvement of learning and memory deficits, a significant decrease in cerebral amyloid- $\beta(A \beta)$ levels and plaque burden, a marked alleviation in neuroinflammation, and a profound reduction in synaptic defects in the APPswe/PS1dE9 mice. Furthermore, amyloid precursor protein (APP) processing was strongly suppressed with Z-GS treatment by inhibiting beta-site APP cleaving enzyme 1 (BACE1) protein expression to produce $A \beta$ in the APPswe/PS1dE9 mice. In addtion, Z-GS modulated anti-inflammatory pathway via decreasing phosphorylation of inhibitor of nuclear factor-kappa B alpha (IKBa) and p65, which was achieved by inhibiting the activation of TLR4.

\section{Conclusions}

Collectively, Our results demonstrate that Z-GS protects neurons against inflammatory damage in $A D$, and this neuroprotective property involves the TLR4/NF-KB signaling pathway.

\section{Background}

Alzheimer's disease (AD) is the most common neurodegenerative disorder characterized by progressive cognitive dysfunction and behavioral impairment. The major pathological hallmarks of AD include neuritic plaques, neurofibrillary tangles, and neuronal loss [1]. Although the exact etiology of $A D$ has not yet been clarified, accumulating evidence has demonstrated that chronic inflammation in the brain plays a crucial role in the pathogenesis of $A D$ [2]. Increased production of amyloid- $\beta(A \beta)$ induces activation of glial cells, expression of pro-inflammatory genes and secretion of inflammatory mediators such as IL-1 $\beta$, IL-6, and TNF-a $[3,4]$. The inflammatory mediators secreted by glial cells in turn promote the expression 
of beta-site APP cleaving enzyme 1 (BACE1) and raise the production of $A \beta$, thus contributing to a vicious cycle [5]. Recent studies have indicated that neuroinflammation in the AD directly intervenes synaptic activity and neurotransmission of neurons, leading to cognitive defects [6]. Therefore, elucidating the pathogenic mechanism of chronic inflammation in $A D$ will conduce to the role of anti-inflammatory strategies in AD treatment.

Toll-like receptor 4 (TLR4) is a transmembrane protein that belongs to the pattern recognition receptor family, which plays a key role in the innate immune system. In addition to pathogen-associated molecular patterns, TLR4 can be activated by certain endogenous ligands produced due to tissue injury and/or inflammation [7]. Following binding with A $\beta$, TLR4 can promote nuclear factor-kappa B (NF-kB) complex activation and downstream events that participate in the transcriptional expression of NLRP3 and IL-1 $\beta$ [8]. Notably, it has been reported that the rs $4986790 \mathrm{G}$ coding variant in the TLR4 gene appears to reduce $A D$ risk through the modulation of IL-1 $\beta$ synthesis and secretion in the presymptomatic phase of the disease [9]. Moreover, previous study has shown that $A \beta$ oligomer or fibrillar $A \beta$ increases the expression of TLR4 and induces microglial activation, leading to cognitive impairment [10]. The inhibition of TLR4 through function blocking antibodies or siRNA knockdown was demonstrated to prevent fibrillar A $\beta$ induced IL- 6 and TNF-a production [11]. Taken together, these findings suggest that the activation of TLR4 is likely involved in chronic inflammation in AD.

Z-Guggulsterone (Z-GS, as shown in Fig. 1) is the main biologically active principle derived from the resin of commiphora mukul. Due to its anti-inflammatory potential, Z-GS has been used as an effective herbal medication for the treatment of arthritis, obesity, atherosclerosis, and cancer [12]. In recent years, the neuroprotective property of Z-GS has drawn increasing interest. Previous study has reported that Z-GS attenuates astrocytes-mediated neuroinflammation and protects the brain from acute damage induced by ischemia/reperfusion [13]. In addition, Z-GS was found to improve the scopolamine-induced memory impairments and ameliorate multiple sclerosis-related motor neuron dysfunctions in rodent models [14, 15]. Evidences have indicated that Z-GS exerts potent anti-inflammatory activity through inhibiting TLR4/NF-KB signaling pathway and its dependent inflammatory cytokines [13, 16-18]. However, whether Z-GS treatment may regulate inflammatory cascades associated with AD remains unknown.

Given the chronic inflammation in the AD brain, it is therefore of interest to explore whether Z-GS treatment can reverse cognitive deficits and neuroinflammation in transgenic mouse models of $A D$. The present study was undertaken to investigate the disease-modifying effect of chronic Z-GS administration and its underlying mechanisms in the APPswe/PS1dE9 mice.

\section{Methods}

\section{Animals and treatment}

As our previous studies have revealed [19, 20], the APPswe/PS1dE9 double-transgenic mice harboring human APPswe (Swedish mutations K594N/M595L) and presenilin-1 with deletion of the exon 9 (PS1- 
dE9) were purchased from Beijing HFK Bio-technology Co., Ltd (Beijing, China). The 9-month-old male APPswe/PS1dE9 mice with typical AD cognitive and pathological impairment characteristics and their matched non-transgenic wild-type littermates were randomly divided into four groups ( $n=7$, each group): vehicle-treated wild-type mice, Z-GS-treated wild-type mice, vehicle-treated APPswe/PS1dE9 mice, and ZGS-treated APPswe/PS1dE9 mice. Male mice were specially selected to exclude possible effects of estrogen [21]. Z-GS (Santa Cruz, CA, USA) was dissolved in dimethylsulfoxide (DMSO) and stored at $-20^{\circ} \mathrm{C}$, final concentration of DMSO is less than $5 \%$ (the concentration was adjusted with normal saline). Each mouse with Z-GS treatment received Z-GS at a dosage of $30 \mathrm{mg} / \mathrm{kg}$ body weight via intraperitoneal injection once per day for 12 weeks, whereas each mouse with vehicle treatment was injected only with an equal volume of $5 \% \mathrm{DMSO} /$ saline solution as control. The dosage of Z-GS was based upon previous studies in which $30 \mathrm{mg} / \mathrm{kg}$ Z-GS administered to mice yielded sufficient concentrations in the brain [13, $14,16]$. Animals were housed under a 12 hours light/dark cycle and had free access to food and water. Body weights and overall general health of all mice were recorded daily. All procedures with mice were conducted under protocols approved by the Institutional Animal Experiment Committee of the Chinese PLA General Hospital and in accordance with the Hospital Policies on the Use and Care of Animals.

\section{Behavioral testing}

After 12 weeks of drug treatment, all mice were assessed for spatial learning and memory using Morris water maze test as the previous studies $[19,20]$. In brief, the poor performance in the acquisition test and probe trial resulted from cognitive deficits rather than noncognitive factors was initially identified with a visible platform test. For evaluating the spatial learning performance, a acquisition test (4 trials per day for 6 consecutive days) was performed. The hidden platform (10 cm in diameter) was kept within the same target quadrant and submerged $1.5 \mathrm{~cm}$ below the water surface. The time needed for an individual mouse to reach the hidden platform was recorded as the escape latency. On completion of the acquisition test, a probe trial was conducted 24 hours later to measure the spatial memory. The platform was removed and mice were given 60 seconds to swim freely in the pool. The percentage of time that each mouse spent in the target quadrant previously containing the hidden platform was recorded as a spatial memory score.

\section{Brain tissue preparation}

After behavioral testing, all mice were deeply anesthetized and transcardially perfused with ice-cold physiological saline. Brains were quickly removed and dissected into two hemispheres through the midsagittal plane. One hemisphere was placed in $4 \%$ paraformaldehyde, followed by embedding in paraffin for immunohistochemical analysis. The entire cerebral cortex and hippocampus were isolated from the remaining hemisphere and stored at $-80^{\circ} \mathrm{C}$ for further biochemical measurements.

\section{Immunohistochemical analysis}

Immunohistochemical analysis was performed as described in our previous studies $[19,20]$. Brain sections were deparaffinated and rehydrated. The following treatments were used for antigen retrieval: proteinase $\mathrm{K}(0.2 \mathrm{mg} / \mathrm{ml})$ for $\mathrm{A} \beta$ staining, and $0.01 \mathrm{M}$ sodium citrate solution for astrocyte and microglia 
staining. After nonspecific binding sites were blocked, the sections were incubated with mouse monoclonal anti-A $\beta$ antibody (DE2) for the detection of human A $\beta 1-16$ (1:200, Millipore, Billerica, MA, USA), mouse monoclonal anti-GFAP antibody targeting astrocytes (1:500; Abcam, Cambridge, MA, USA), and rabbit monoclonal anti-lba1 antibody targeting microglia (1:1,000; Abcam, Cambridge, MA, USA) overnight at $4^{\circ} \mathrm{C}$. Primary antibodies were detected with a horseradish peroxidase-conjugated secondary antibody and visualized with a stable diaminobenzidine solution (Vector Laboratories, Burlingame, CA, USA). $1 \%$ Thioflavin S (Sigma-Aldrich, St Louis, MO, USA) was used for fibrillar plaques staining, and green fluorescence from plaques was visualized by fluorescence microscopy. For quantification, microscopic images were collected to calculate the percentages of area occupied by Aß1-16-positive plaques, Thioflavin S-positive plaques, GFAP-positive astrocytes, and Iba1-positive microglia by using the Image-Pro Plus imaging software (Media Cybernetics, Bethesda, MD, USA).

\section{Quantification of cerebral $A \beta$ and inflammatory mediators}

The levels of cerebral $A \beta$ and inflammatory mediators were analyzed by ELISA according to manufacturer's directions. In brief, the cerebral cortex and hippocampus homogenates were sequentially extracted firstly in Tris-buffered saline (TBS) for the soluble $A \beta$ fractions and then in formic acid (FA) for the insoluble $A \beta$ fractions. The soluble and insoluble $A \beta$ levels were determined by the commercial human A $\beta 1-40$ and A $\beta 1-42$ ELISA kits (Invitrogen, Camarillo, CA, USA). The commercial human amyloid- $\beta$ oligomers ELISA kit was used to measure soluble $A \beta$ oligomer levels (Immuno Biochemical Laboratories, Gunma, Japan). For evaluating cerebral inflammation, mouse brain tissue was initially homogenized in $2 \mathrm{ml}$ of $10 \mathrm{mM}$ phosphate buffer. The levels of interleukin-1 $\beta$ (IL-1 $\beta$ ), interleukin-6 (IL-6), and tumor necrosis factor- $a$ (TNF-a) were quantified using the corresponding ELISA kits (Nanjing Jiancheng Biochemistry Co., Nanjing, China).

\section{Western blot analysis}

RIPA extracts from brain homogenates were used for western blot analyses as reported in our recent studies $[19,20]$. Briefly, equal amounts of protein (20 $\mu \mathrm{g}$ of protein each sample) were separated by polyacrylamide gel electrophoresis (PAGE), and then transferred to polyvinylidiene diflouride (PVDF) membranes. After blockage of nonspecific binding sites, the membranes were probed with the following antibodies overnight at $4^{\circ} \mathrm{C}$ : rabbit anti-TLR4 antibody (1:1,000; Abcam), mouse anti-IKBa antibody (1:1000; Cell Signaling Technology), mouse anti-p-IкBa (Ser32/36) antibody (1:500; Santa Cruz Biotechnology), mouse anti-NF-KB p65 antibody (1:1000; Cell Signaling Technology), rabbit anti-NF-KB pp65 (Ser536) antibody (1:1000; Cell Signaling Technology), mouse anti-APP antibody (1:500; Millipore), mouse anti-human sAPPa antibody (1:100; Immuno-Biological Laboratories), mouse anti-human sAPP $\beta$ antibody (1:100; Immuno-Biological Laboratories), rabbit anti-ADAM10 antibody (1:2,000; Abcam), rabbit anti-BACE1 antibody (1:1,000; Abcam), rabbit anti-PS1 antibody (1:500; Abcam), rabbit anti-NEP antibody (1:1,000; Millipore), goat anti-IDE antibody (1:500; Sigma-Aldrich), rabbit anti-SYP antibody (1:1,000; Cell Signaling Technology), rabbit anti-PSD95 antibody (1:500; Abcam), and mouse anti- $\beta$-actin antibody (1:5,000; Sigma-Aldrich). Membranes were washed for 3 times with $1 \times$ TBST, followed by incubation with horseradish peroxide-conjugated second antibodies (1:5,000; Santa Cruz Biotechnology) for 2 hours at 
room temperature. Finally, the bands were scanned by an automated image analysis system (Bio-Rad, Hercules, CA, USA).

\section{Statistical analysis}

All statistical analyses were performed using SPSS 17.0 software (SPSS Inc., Chicago, IL, USA). The group differences in the escape latency during Morris water maze test were calculated using two-way analysis of variance (ANOVA) with repeated measures followed by post hoc LSD test for multiple comparisons. All other data were analyzed with One-way ANOVA followed by post hoc LSD test or student's $t$ test. All data were presented as mean \pm SEM. A value of $p<0.05$ was considered statistically significant.

\section{Results}

\section{Z-GS treatment ameliorates cognitive deficiencies of APPswe/PS1dE9 mice}

Morris water maze test was applied to assess spatial learning and memory performances of all mice. In visible platform test, the wild-type and transgenic mice exhibited similar swimming speeds and escape latencies (data not shown; $p>0.05$ ), indicating that the vision or locomotion of mice was not affected by Z-GS treatment. As shown in Fig. 2A, at 12 months of age, the vehicle-treated APPswe/PS1dE9 mice displayed impaired spatial learning in the acquisition trials, as indicated by greater escape latencies to target the hidden platform compared with the wild-type mice (ANOVA with repeated measures; group effect: $F(1,12)=45.942, p<0.001$; training day effect: $F(5,60)=193.983, p<0.001$; group $\times$ training day interaction: $F(5,60)=1.82, p>0.05)$. In contrast, Z-GS treatment markedly improved the spatial learning deficiencies in the APPswe/PS1dE9 mice, where Z-GS treatment significantly reduced the escape latencies of transgenic mice (ANOVA with repeated measures; group effect: $F(1,12)=17.059, p<0.01$; training day effect: $F(5,60)=155.859, p<0.001$; group $\times$ training day interaction: $F(5,60)=0.519, p>$ $0.05)$. After the acquisition trials, the probe test was performed to assess spatial memory of all mice. As shown in Fig. 2B, APPswe/PS1dE9 mice treated with vehicle showed impaired spatial memory, as evidenced by less time spent in the target quadrant compared with the wild-type mice $(p<0.01)$. However, Z-GS treatment remarkably ameliorates the impaired spatial memory in the APPswe/PS1dE9 mice, with longer time in the target quadrant than the vehicle-treated transgenic controls $(p<0.01)$. Collectively, these results demonstrate that TLR4 inhibition with Z-GS treatment significantly improves cognitive defects in the APPswe/PS1dE9 mice.

\section{Z-GS treatment reduces cerebral $A \beta$ levels and plaque burden in APPswe/PS1dE9 mice}

After Z-GS treatment, we measured cerebral $A \beta$ levels and plaque burden in the transgenic mouse brain by using ELISA and immunohistochemistry. As shown in Fig. 3, the levels of soluble and insoluble A $1-$ 
40 (Fig. 3A and C) and A 1 1-42 (Fig. 3B and D) were dramatically declined in the cerebral cortex and hippocampus of APPswe/PS1dE9 mice treated with Z-GS compared with the vehicle-treated transgenic control group (respectively; $p<0.001$ ). Moreover, Z-GS treatment markedly reduced the levels of soluble $A \beta$ oligomers in the cerebral cortex and hippocampus of APPswe/PS1dE9 mice (Fig. 3E; $p<0.001$ ). In accordance with these results, the proportions of area occupied by diffused and fibrillar plaques were found to be remarkably decreased in the cerebral cortex and hippocampus of Z-GS-treated APPswe/PS1dE9 mice compared to the vehicle-treated transgenic controls (Fig. $4 ; p<0.001$ ). Taken together, these findings demonstrate inhibition of TLR4 by Z-GS treatment is dramatically effective in reducing $A \beta$ production and deposition in the brains of APPswe/PS1dE9 mice.

\section{Z-GS treatment suppresses BACE1-mediated amyloidogenic pathway in APPswe/PS1dE9 mice}

In order to investigate the exact mechanisms underlying $A \beta$ reduction with Z-GS treatment, the effects of Z-GS on the expression of full-length APP, soluble APP-alpha (SAPPa, a major product of APP cleaved by a-secretase), soluble APP-beta (sAPP $\beta$, a major product of APP cleaved by $\beta$-secretase), the different proteases (ADAM10, BACE1, and PS1) involved in APP processing as well as neprilysin (NEP) and insulindegrading enzyme (IDE) involved in $A \beta$ degradation were assessed by western blot. As shown in Fig. 5D, the levels of SAPP $\beta$ were dramatically reduced in the cerebral cortex and hippocampus of APPswe/PS1dE9 mice treated with Z-GS compared to vehicle controls $(p<0.001)$. However, no significant difference in the expression of full-length APP or SAPPa in both regions was observed between the vehicle- and Z-GS-treated APPswe/PS1dE9 mice (Fig. 5B and C; $p>0.05$ ). Consistently, the expression of BACE1 was remarkably decreased in the cerebral cortex and hippocampus of Z-GS-treated APPswe/PS1dE9 mice relative to vehicle-treated transgenic controls (Fig. 5E; $p<0.01$ ), whereas ADAM10 or PS1 levels in both regions of APPswe/PS1dE9 mice were not affected by Z-GS treatment (Fig. 5F; $p>$ 0.05), indicating Z-GS inhibits $\beta$-secretase cleavage of APP in the brains of APPswe/PS1dE9 mice. In addtion, the levels of NEP and IDE protein expression showed no difference in the cerebral cortex and hippocampus among the four groups of mice ( $p>0.05$; data not shown). Together, these data prove that Z-GS-mediated decrease of $A \beta$ production may result form its inhibition of amyloidogenic pathway, rather than enhanced non-amyloidogenic pathway and increased A $\beta$ degradation in the APPswe/PS1dE9 mouse brain.

\section{Z-GS treatment alleviates cerebral inflammation and gliosis in APPswe/PS1dE9 mice}

Given the critical role of cerebral inflammation in the pathogenesis of $A D$ [2], we evaluated inflammatory mediators in the brains from wild-type and transgenic mice. Compared with the wild-type group, the levels of IL-1 $\beta, \mathrm{IL}-6$, and TNF- $\alpha$ in the cerebral cortex and hippocampus were significantly increased in the vehicle-treated APPswe/PS1dE9 mice (Fig. 6A-C; $p<0.001$ ). Nevertheless, Z-GS treatment remarkably reduced the expression of these mediators in both regions of APPswe/PS1dE9 mice (Fig. 6A-C; $p<0.01$ ). 
To further confirm the beneficial effect of Z-GS treatment on the inflammatory response, we measured activated astrocyte and microglial reactivity in the brains of APPswe/PS1dE9 mice. An anti-glial fibrillary acidic protein (GFAP) antibody for astrocytes and an anti-ionized calcium binding adaptor molecule 1 (Iba1) antibody for microglia were applied in immunostaining serial sections (Fig. 7A-H). As shown in Fig. $7 \mathrm{I}$ and $\mathrm{J}$, the percentages of brain area occupied by GFAP-immunoreactive astrocytes and Iba1immunoreactive microglia were remarkably increased in the cerebral cortex and hippocampus of vehicletreated APPswe/PS1dE9 mice relative to wild-type mice $(p<0.01)$. However, Z-GS treatment significantly decreased the densities of GFAP-positive astrocytes and Iba1-positive microglia in both regions of APPswe/PS1dE9 mice (Fig. $7 \mathrm{I}$ and J; $p<0.01$ ). These data suggest that TLR4 inhibition with Z-GS treatment markedly alleviates inflammatory response and glial activation in the brains of APPswe/PS1dE9 mice.

\section{Z-GS treatment attenuates synaptopathy in APPswe/PS1dE9 mice}

Considering synaptic dysfunction closely associated with AD cognitive defects [22], we identified the effect of Z-GS treatment on synaptopathy. The expression of synaptophysin (SYP, a presynaptic marker) and postsynaptic density 95 (PSD95, a postsynaptic marker) was examined in each mouse brain. Compared with wild-type mice, the levels of SYP and PSD95 were documented to be significantly declined in the cerebral cortex and hippocampus of vehicle-treated APPswe/PS1dE9 mice (Fig. 8B and C; $p<0.01)$. In contrast, a dramatical elevation in the SYP and PSD95 expression was induced with Z-GS treatment in both areas of APPswe/PS1dE9 mice (Fig. 8B and C; $p<0.01$ ). These results demonstrate that TLR4 inhibition with Z-GS treatment remarkably ameliorates synaptic dysfunction in the brains of APPswe/PS1dE9 mice.

\section{Z-GS treatment inhibits TLR4/NF-KB pathway in APPswe/PS1dE9 mice}

As shown in Fig. 9, the levels of TLR4, p-IKBa, and p-p65 were significantly increased in the cerebral cortex and hippocampus of the vehicle-treated APPswe/PS1dE9 mice compared with the wild-type mice ( $p<$ 0.01). After Z-GS treatment, the expression of TLR4, p-IKBa, and p-p65 was markedly decreased in both regions of APPswe/PS1dE9 mice $(p<0.01)$. However, no significant differences in the expression of TLR4, $\mathrm{p}$-IKBa, and $\mathrm{p}-\mathrm{p} 65$ were documented between vehicle- and Z-GS-treated wild-type mice $(p>0.05)$. These data indicate that Z-GS treatment strongly inhibits TLR4/NF-KB signaling pathway in the brains of APPswe/PS1dE9 mice.

\section{Chronic administration of Z-GS has no observable side effects on mice}


Throughout the study, no abnormal macroscopic effects were detected on the overall general health of animals treated with vehicle and Z-GS. Furthermore, we did not find significant differences in body weight and whole brain weight among the four groups (data not shown; $p>0.05$ ). In addtion, similar performance in behavioral tests and biochemical responses were observed between vehicle- and Z-GStreated wild-type mice (Figs. 2-9). Based on these data, it appears that chronic treatment with Z-GS has no observable adverse effects on mice. However, further in vivo studies investigating acute and chronic toxicities of Z-GS are warranted to confirm our findings.

\section{Discussion}

In the present study, we documented that Z-GS exerted protective effects on cognitive and neuropathological impairments in the transgenic mouse models of $A D$, which was manifested in improved cognitive defects, decreased $A \beta$ production and deposition, alleviated inflammatory response and glial activation, and reduced synaptic loss. The mechanism is probably attributed to down-regulation of BACE1 expression through inhibiting the TLR4/NF-KB signaling pathway.

The detrimental role of chronic inflammation together with the neurodegenerative events involved in $A D$ has been widely explored for new potential therapeutic approaches. Due to the known contribution of innate immune and inflammatory response, TLR4 has received increased attention and thus representing a promising target for inflammation-based AD therapy [23]. A number of studies have shown that the expression of TLR4 is abnormally increased in the brains of AD patients and mice with AD-like pathology [24-26]. High TLR4 immunoreactivity was observed in glial cells surrounding A $\beta$ plaques in postmortem brains of $A D$ patients [24], and significantly higher levels of pro-inflammatory cytokines have been detected in the brains of APP/PS1 mice [27]. Moreover, oligomeric and fibrillar A $\beta$ peptide promoted the discharge of pro-inflammatory and neurotoxic cytokines IL-1 $\beta$, IL-6, and TNF- $a$ by microglia and astrocytes in a TLR4-dependent manner [28]. Notably, intracerebroventricular injection of A $\beta$ induced an inflammatory response leading to neuronal death, synaptic loss, and cognitive impairment in wild-type mice but not in TLR4 knockout mice [10]. However, TLR4 antagonists were found to abolish A $\beta$ oligomerinduced astrocyte activation, neuronal cell death, and long-term potentiation deficit in hippocampal slices [29]. Collectively, these findings suggest that specific inhibition of TLR4 is likely a potential therapeutic strategy for AD. In accordance with the previous studies [26, 30], we found that TLR4 expression levels were significantly increased in the brains of APPswe/PS1dE9 mice, indicating TLR4 signaling pathway was excessively activated in the brains of mice with AD-like pathology. Nevertheless, chronic administration of Z-GS in the APPswe/PS1dE9 mice strongly inhibited TLR4 expression down to the basal levels of wild-type mice. Furthermore, Z-GS dramaticaly inhibited the over-phosphorylation of IKBa and $\mathrm{p} 65$ and markedly attenuated inflammatory response by decreasing pro-inflammatory cytokines IL$1 \beta, I L-6$, and TNF- $\alpha$ in the brains of APPswe/PS1dE9 mice. Taken together, these results demonstrate that the neuroprotective role of Z-GS against chronic inflammation in APPswe/PS1dE9 mice is probably attributed to its anti-inflammatory capability by suppressing the TLR4/ NF-KB pathway. 
Many lines of evidence support that $A \beta$ peptides and amyloid plaques play important roles in the pathogenesis of $A D$ [31]. BACE1 is required for the generation of $A \beta$, which aggregates into bioactive conformational species and likely initiates toxicity in $A D$. Given the increased concentration and activity of BACE1 in $A D$ brain, it is thereby making BACE1 a prime target for slowing down $A \beta$ production in $A D$ $[32,33]$. In the present study, Z-GS treatment were found to markedly reduce the production and deposition of $A \beta$ in the APPswe/PS1dE9 mice relative to the transgenic controls, showing that $A \beta-$ initiated pathological cascades in AD might be restrained by Z-GS treatment. To further elucidate the mechanisms responsible for Z-GS-modulated decrease in $A \beta$ generation and accumulation, we measured the levels of full-length APP, the major secretases (ADAM10, BACE1, and PS1) involved in APP metabolism, and enzymes for A $\beta$ degradation (NEP and IDE). We documented that Z-GS treatment significantly depressed the expression of BACE1 and the release of its cleavage product SAPP $\beta$ in the brains of APPswe/PS1dE9 mice, suggesting that Z-GS treatment was likely to suppress amyloidogenic APP pathway mediated by BACE1 in AD brain. By contrast, the expression levels of full-length APP, PS1, ADAM10 and its cleavage product SAPPa as well as NEP and IDE were not affected by Z-GS treatment in the APPswe/PS1dE9 mice, indicating that the $A \beta$ decease was not regulated by the synthesis and $a-$ cleavage proteolytic processing of APP and degradation of $A \beta$. Although there is no evidence that TLR4 directly regulates BACE1 expression, NF-KB signaling has been shown to enhance BACE1 expression in the brain $[34,35]$. Considering the activation of NF-KB signaling is a downstream event provoked by TLR4, it appears that the $A \beta$ reduction mediated by BACE1 with Z-GS treatment in the brains of APPswe/PS1dE9 mice may be attributed to its selective inhibition of TLR4/NF-KB pathway.

There has been abundant research in recent years to explain the role of gliosis in the development of $A D$ [36]. It has been demonstrated that $A \beta$ plaque formation and reactive gliosis are required for induction of cognitive deficits in mouse models of AD [37]. Moreover, presence of activated glias and inflammationrelated mediators association with the $A \beta$ lesions was shown in the brains of patients with $A D$ pathology [38]. Compared with the transgenic controls, we found that Z-GS treatment remarkably alleviated the hyperplasia of astrocytes and microglia in the APPswe/PS1dE9 mice in the present study. A number of studies have implied that $A \beta$ peptides induce the proliferation and activation of astrocytes and microglia $[39,40]$. It seems that the dramatical decrease of reactive gliosis observed in the brains of APPswe/PS1dE9 mice treated with Z-GS might be secondary to the reduction of A $\beta$ assemblage. However, there is increasing evidence showing that TLR4/NF-KB signaling plays a crucial role in astrocyte and microglial activation [41, 42]. Therefore, the significant reduction of activated astrocytes and microglia in the Z-GS-treated APPswe/PS1dE9 mice may result from the inhibition of TLR4/NF-KB pathway rather than the decline of $A \beta$ accumalation.

Synapse loss and synaptic dysfunction are critical processes involved in the pathophysiology of $A D$, which are more directly tied to the severity of cognitive defects [43]. Compelling evidence suggests that soluble $A \beta$ peptide oligomers induce synaptic loss in AD [44]. Moreover, it has been reported that hippocampal accumulation of $A \beta$ is responsible for reducing dendritic protein MAP2 and dendritic spines and hippocampal based learning and memory impairments [45]. In addition, IL-1 $\beta$ and TNF- $\alpha$ were found to suppress hippocampal long-term potentiation directly at the synapse [46]. Accordingly, the 
mechanisms underlying the restoration of impaired synaptic plasticity and integrity in the Z-GS-treated mouse models of $A D$ in the present study is probably attributed to its multiple effects in reducing $A \beta$ production and neuroinflammation.

Cognitive impairment is the major clinical hallmark of $A D$ and gradually worsens as the disease progresses [47]. There is an urgent need to develop therapeutic strategies that may alleviate or reverse the symptoms of cognitive deficits in AD. Consistent with our previous studies [19, 20], the 12 months old APPswe/PS1dE9 mice treated with vehicle showed marked spatial learning and memory impairments compared with wild-type mice. Nevertheless, TLR4 inhibition with Z-GS treatment significantly improved the cognitive defects in the mouse models with AD-like pathology. Notably, a number of studies have exhibited that suppression of TLR4 activation attenuates $A \beta$ or lipopolysaccharide-induced cognitvie impairment [48, 49]. In accordance with these findings, we indicated that Z-GS treatment dramatically inhibited TLR4 activation, contributing to $A \beta$ reduction, neuroinflammtion alleviation, and synaptic dysfunction recovery in the brains of APPswe/PS1dE9 mice. Therefore, the beneficial effect of TLR4 inhibition with Z-GS treatment on cognitive improvement in the mouse models of $A D$ is likely result from the amelioration of diverse neuropathological damages.

\section{Limitations}

There are three limitations of this study. First, cognitive impairment is the major clinical hallmark of AD and is associated with a variety of pathological processes. Our study showed that Z-GS improved cognitive deficits in APPswe/PS1dE9 mice by reducing the production and deposition of $A \beta$, alleviating neuroinflammation and gliosis, and repairing synaptic dysfunction. However, whether Z-GS can improve other pathological processes related to $A D$ cognitive defects remains to be further investigated. Furthermore, the exact pathogenesis of $A D$ has not yet been fully elucidated. Our study found that Z-GS could ameliorate the cognitive deficits and pathological impairments of $A D$ transgenic mice through modulating the TLR4/NF-KB signaling pathway. Nevertheless, the underlying mechanism of Z-GS in the treatment of $A D$ is still worth exploring. In addition, there is an increasing interest in the neuroprotective effects of existing natural and synthetic compounds on AD. Although these compounds including Z-GS that exert their protective effects in vitro or in mice, there is no translation to AD patients. Therefore, further trials are clearly necessary to establish the clinical efficacy of Z-GS. These three limitations set the ground for future studies.

\section{Conclusions}

In conclusion, our results proved that Z-GS treatment strongly inhibited TLR4/NF-KB signaling pathway, which led to improvement of cognitive dysfunction, decrease of $A \beta$ production, amelioration of inflammatory injury and gliosis, and attenuation of synaptopathy in APPswe/PS1dE9 mice. Notably, we found that Z-GS treatment potently suppressed amyloidogenic APP processing by inhibiting BACE1 expression to preclude $A \beta$ production. These findings suggest Z-GS is probably a novel diseasemodifying therapeutic approach for $A D$. 


\section{Abbreviations}

$A \beta$, amyloid- $\beta$; AD, Alzheimer's disease; ADAM10, a disintegrin and a metalloproteinase 10; APP, amyloid precursor protein; BACE1, beta-site APP cleaving enzyme 1; FA, formic acid; GFAP, glial fibrillary acidic protein; Iba1, ionized calcium binding adaptor molecule 1; IDE, insulin-degrading enzyme; IKBa, inhibitor of nuclear factor-kappa B alpha; IL-1 $\beta$, interleukin 1 beta; IL-6, interleukin 6; NF-KB, nuclear factor-kappa B; NLRP3, NACHT, LRR, and PYD domains-containing protein 3; NEP, neprilysin; PAGE, polyacrylamide gel electrophoresis; PS1, presenilin-1; PSD95, postsynaptic density 95; PVDF, polyvinylidiene diflouride; sAPPa, soluble APP-alpha; SAPP $\beta$, soluble APP-beta; SYP, synaptophysin; TBS, Tris-buffered saline; TNF-a, tumor necrosis factor alfa; TLR4, toll-like receptor 4

\section{Declarations}

\section{Acknowledgements}

The authors thank Dr. Shuo Liu for assistance with immunohistochemical examination.

\section{Funding}

This work was supported by research grant from the National Natural Science Foundation of China (Nos. 81801044).

\section{Availability of data and materials}

The datasets used and analyzed in this study are available from the corresponding author on reasonable request.

\section{Author Contributions}

Conceived and designed the experiments: Wei $\mathrm{C}$ and Fan J. Performed the experiments: Liu J, Yang Y, Guo Y, Shang Y, Zhou B. Analyzed the data: Wei C and Fan J. Wrote the manuscript: Wei C and Fan J.

\section{Ethics approval}

This study involved the use of animals. All procedures were reviewed and approved by the Institutional Animal Experiment Committee of the Chinese PLA General Hospital and in accordance with the Hospital Policies on the Use and Care of Animals.

\section{Consent for publication}

Not applicable.

\section{Competing interests}

The authors have declared no conflicts of interest. 


\section{References}

1. Querfurth HW, LaFerla FM. Alzheimer's disease. N Engl J Med. 2010;362:329-44.

2. Heppner FL, Ransohoff RM, Becher B. Immune attack: the role of inflammation in Alzheimer disease. Nat Rev Neurosci. 2015;16:358-72.

3. Xu PX, Wang SW, Yu XL, Su YJ, Wang T, Zhou WW, et al. Rutin improves spatial memory in Alzheimer's disease transgenic mice by reducing Abeta oligomer level and attenuating oxidative stress and neuroinflammation. Behav Brain Res. 2014;264:173-80.

4. McGeer PL, McGeer EG. Inflammation, autotoxicity and Alzheimer disease. Neurobiol Aging. 2001;22:799-809.

5. Newcombe EA, Camats-Perna J, Silva ML, Valmas N, Huat TJ, Medeiros R. Inflammation: the link between comorbidities, genetics, and Alzheimer's disease. J Neuroinflammation. 2018;15:276.

6. Calsolaro V, Edison P. Neuroinflammation in Alzheimer's disease: Current evidence and future directions. Alzheimers Dement. 2016;12:719-32.

7. Molteni M, Gemma S, Rossetti C. The Role of Toll-Like Receptor 4 in Infectious and Noninfectious Inflammation. Mediators Inflamm. 2016;2016:6978936.

8. Yang J, Wise L, Fukuchi KI. TLR4 Cross-Talk With NLRP3 Inflammasome and Complement Signaling Pathways in Alzheimer's Disease. Front Immunol. 2020;11:724.

9. Miron J, Picard C, Lafaille-Magnan ME, Savard M, Labonte A, Breitner J, et al. Association of TLR4 with Alzheimer's disease risk and presymptomatic biomarkers of inflammation. Alzheimers Dement. 2019;15:951-60.

10. Balducci C, Frasca A, Zotti M, La Vitola P, Mhillaj E, Grigoli E, et al. Toll-like receptor 4-dependent glial cell activation mediates the impairment in memory establishment induced by beta-amyloid oligomers in an acute mouse model of Alzheimer's disease. Brain Behav Immun. 2017;60:188-97.

11. Trotta T, Porro C, Calvello R, Panaro MA. Biological role of Toll-like receptor-4 in the brain. J Neuroimmunol. 2014;268:1-12.

12. Yamada T, Sugimoto K. Guggulsterone and Its Role in Chronic Diseases. Adv Exp Med Biol. 2016;929:329-61.

13. Liu T, Liu M, Zhang T, Liu W, Xu H, Mu F, et al. Z-Guggulsterone attenuates astrocytes-mediated neuroinflammation after ischemia by inhibiting toll-like receptor 4 pathway. J Neurochem. 2018;147:803-15.

14. Chen Z, Huang C, Ding W. Z-Guggulsterone Improves the Scopolamine-Induced Memory Impairments Through Enhancement of the BDNF Signal in C57BL/6J Mice. Neurochem Res. 2016;41:3322-32.

15. Kumar N, Sharma N, Khera R, Gupta R, Mehan S. Guggulsterone ameliorates ethidium bromideinduced experimental model of multiple sclerosis via restoration of behavioral, molecular, neurochemical and morphological alterations in rat brain. Metab Brain Dis. 2021;36:911-25.

16. Huang C, Wang J, Lu X, Hu W, Wu F, Jiang B, et al. Z-guggulsterone negatively controls microgliamediated neuroinflammation via blocking IkappaB-alpha-NF-kappaB signals. Neurosci Lett. 
2016;619:34-42.

17. Zhang JH, Shangguan ZS, Chen C, Zhang HJ, Lin Y. Anti-inflammatory effects of guggulsterone on murine macrophage by inhibiting LPS-induced inflammatory cytokines in NF-kappaB signaling pathway. Drug Des Devel Ther. 2016;10:1829-35.

18. Kim DG, Bae GS, Jo IJ, Choi SB, Kim MJ, Jeong JH, et al. Guggulsterone Attenuated Lipopolysaccharide-Induced Inflammatory Responses in Mouse Inner Medullary Collecting Duct-3 Cells. Inflammation. 2016;39:87-95.

19. Wei C, Zhang W, Zhou Q, Zhao C, Du Y, Yan Q, et al. Mithramycin A Alleviates Cognitive Deficits and Reduces Neuropathology in a Transgenic Mouse Model of Alzheimer's Disease. Neurochem Res. 2016;41:1924-38.

20. Wei C, Fan J, Sun X, Yao J, Guo Y, Zhou B, et al. Acetyl-11-keto-beta-boswellic acid ameliorates cognitive deficits and reduces amyloid-beta levels in APPswe/PS1dE9 mice through antioxidant and anti-inflammatory pathways. Free Radic Biol Med. 2020;150:96-108.

21. Xu H, Gouras GK, Greenfield JP, Vincent B, Naslund J, Mazzarelli L, et al. Estrogen reduces neuronal generation of Alzheimer beta-amyloid peptides. Nat Med. 1998;4:447-51.

22. Forner S, Baglietto-Vargas D, Martini AC, Trujillo-Estrada L, LaFerla FM. Synaptic Impairment in Alzheimer's Disease: A Dysregulated Symphony. Trends Neurosci. 2017;40:347-57.

23. Zhou Y, Chen Y, Xu C, Zhang H, Lin C. TLR4 Targeting as a Promising Therapeutic Strategy for Alzheimer Disease Treatment. Front Neurosci. 2020;14:602508.

24. Walter S, Letiembre M, Liu Y, Heine H, Penke B, Hao W, et al. Role of the toll-like receptor 4 in neuroinflammation in Alzheimer's disease. Cell Physiol Biochem. 2007;20:947-56.

25. Miron J, Picard C, Frappier J, Dea D, Theroux L, Poirier J. TLR4 Gene Expression and ProInflammatory Cytokines in Alzheimer's Disease and in Response to Hippocampal Deafferentation in Rodents. J Alzheimers Dis. 2018;63:1547-56.

26. Jin X, Liu MY, Zhang DF, Zhong X, Du K, Qian P, et al. Baicalin mitigates cognitive impairment and protects neurons from microglia-mediated neuroinflammation via suppressing NLRP3 inflammasomes and TLR4/NF-kappaB signaling pathway. CNS Neurosci Ther. 2019;25:575-90.

27. Jin JJ, Kim HD, Maxwell JA, Li L, Fukuchi K. Toll-like receptor 4-dependent upregulation of cytokines in a transgenic mouse model of Alzheimer's disease. J Neuroinflammation. 2008;5:23.

28. Calvo-Rodriguez M, de la Fuente C, Garcia-Durillo M, Garcia-Rodriguez C, Villalobos C, Nunez L. Aging and amyloid beta oligomers enhance TLR4 expression, LPS-induced $\mathrm{Ca}(2+)$ responses, and neuron cell death in cultured rat hippocampal neurons. J Neuroinflammation. 2017;14:24.

29. Hughes C, Choi ML, Yi JH, Kim SC, Drews A, George-Hyslop PS, et al. Beta amyloid aggregates induce sensitised TLR4 signalling causing long-term potentiation deficit and rat neuronal cell death. Commun Biol. 2020;3:79.

30. Cui W, Sun C, Ma Y, Wang S, Wang X, Zhang Y. Inhibition of TLR4 Induces M2 Microglial Polarization and Provides Neuroprotection via the NLRP3 Inflammasome in Alzheimer's Disease. Front Neurosci. 2020;14:444. 
31. Gouras GK, Olsson TT, Hansson O. beta-Amyloid peptides and amyloid plaques in Alzheimer's disease. Neurotherapeutics. 2015;12:3-11.

32. Hampel H, Vassar R, De Strooper B, Hardy J, Willem M, Singh N, et al. The beta-Secretase BACE1 in Alzheimer's Disease. Biol Psychiatry. 2021;89:745-56.

33. Koelsch G. BACE1 Function and Inhibition: Implications of Intervention in the Amyloid Pathway of Alzheimer's Disease Pathology. Molecules 2017, 22.

34. Wang R, Chen S, Liu Y, Diao S, Xue Y, You X, et al. All-trans-retinoic acid reduces BACE1 expression under inflammatory conditions via modulation of nuclear factor kappaB (NFkappaB) signaling. J Biol Chem. 2015;290:22532-42.

35. Du Y, Zhao Y, Li C, Zheng Q, Tian J, Li Z, et al. Inhibition of PKCdelta reduces amyloid-beta levels and reverses Alzheimer disease phenotypes. J Exp Med. 2018;215:1665-77.

36. Merluzzi AP, Carlsson CM, Johnson SC, Schindler SE, Asthana S, Blennow K, et al. Neurodegeneration, synaptic dysfunction, and gliosis are phenotypic of Alzheimer dementia. Neurology. 2018;91:e436-43.

37. Sakakibara Y, Sekiya M, Saito T, Saido TC, lijima KM. Amyloid-beta plaque formation and reactive gliosis are required for induction of cognitive deficits in App knock-in mouse models of Alzheimer's disease. BMC Neurosci. 2019;20:13.

38. Eikelenboom P, van Exel E, Hoozemans JJ, Veerhuis R, Rozemuller AJ, van Gool WA. Neuroinflammation - an early event in both the history and pathogenesis of Alzheimer's disease. Neurodegener Dis. 2010;7:38-41.

39. Hou L, Liu Y, Wang $X, M a H, H e J$, Zhang $Y$, et al. The effects of amyloid-beta42 oligomer on the proliferation and activation of astrocytes in vitro. In Vitro Cell Dev Biol Anim. 2011;47:573-80.

40. Luciunaite A, McManus RM, Jankunec M, Racz I, Dansokho C, Dalgediene I, et al. Soluble Abeta oligomers and protofibrils induce NLRP3 inflammasome activation in microglia. J Neurochem. 2020;155:650-61.

41. Hoogland IC, Houbolt C, van Westerloo DJ, van Gool WA, van de Beek D. Systemic inflammation and microglial activation: systematic review of animal experiments. J Neuroinflammation. 2015;12:114.

42. Rosciszewski G, Cadena V, Murta V, Lukin J, Villarreal A, Roger T, et al. Toll-Like Receptor 4 (TLR4) and Triggering Receptor Expressed on Myeloid Cells-2 (TREM-2) Activation Balance Astrocyte Polarization into a Proinflammatory Phenotype. Mol Neurobiol. 2018;55:3875-88.

43. Chen Y, Fu AKY, Ip NY. Synaptic dysfunction in Alzheimer's disease: Mechanisms and therapeutic strategies. Pharmacol Ther. 2019;195:186-98.

44. Tu S, Okamoto S, Lipton SA, Xu H. Oligomeric Abeta-induced synaptic dysfunction in Alzheimer's disease. Mol Neurodegener. 2014;9:48.

45. Kandimalla R, Manczak M, Yin X, Wang R, Reddy PH. Hippocampal phosphorylated tau induced cognitive decline, dendritic spine loss and mitochondrial abnormalities in a mouse model of Alzheimer's disease. Hum Mol Genet. 2018;27:30-40. 
46. Prieto GA, Tong L, Smith ED, Cotman CW. TNFalpha and IL-1 beta but not IL-18 Suppresses Hippocampal Long-Term Potentiation Directly at the Synapse. Neurochem Res. 2019;44:49-60.

47. Scheltens P, Blennow K, Breteler MM, de Strooper B, Frisoni GB, Salloway S, et al. Alzheimer's disease. Lancet. 2016;388:505-17.

48. Ali W, Ikram M, Park HY, Jo MG, Ullah R, Ahmad S, et al. Oral Administration of Alpha Linoleic Acid Rescues Abeta-Induced Glia-Mediated Neuroinflammation and Cognitive Dysfunction in C57BL/6N Mice. Cells 2020, 9.

49. Wang D, Dong X, Wang B, Liu Y, Li S. Geraniin Attenuates Lipopolysaccharide-Induced Cognitive Impairment in Mice by Inhibiting Toll-Like Receptor 4 Activation. J Agric Food Chem. 2019;67:10079-88.

\section{Figures}

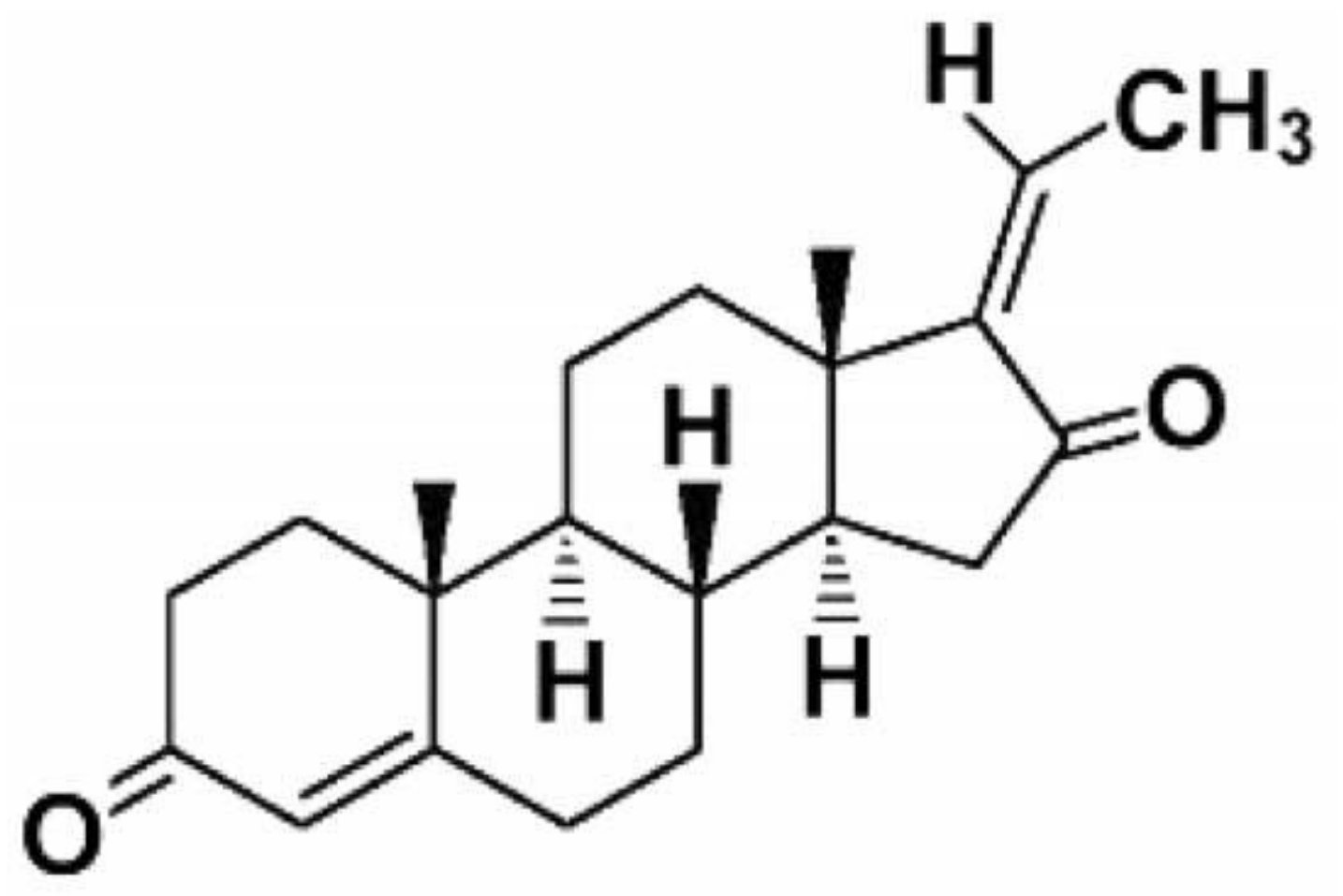

\section{Z-guggulsterone}

\section{Figure 1}

Chemical structure of Z-Guggulsterone (Z-GS). 


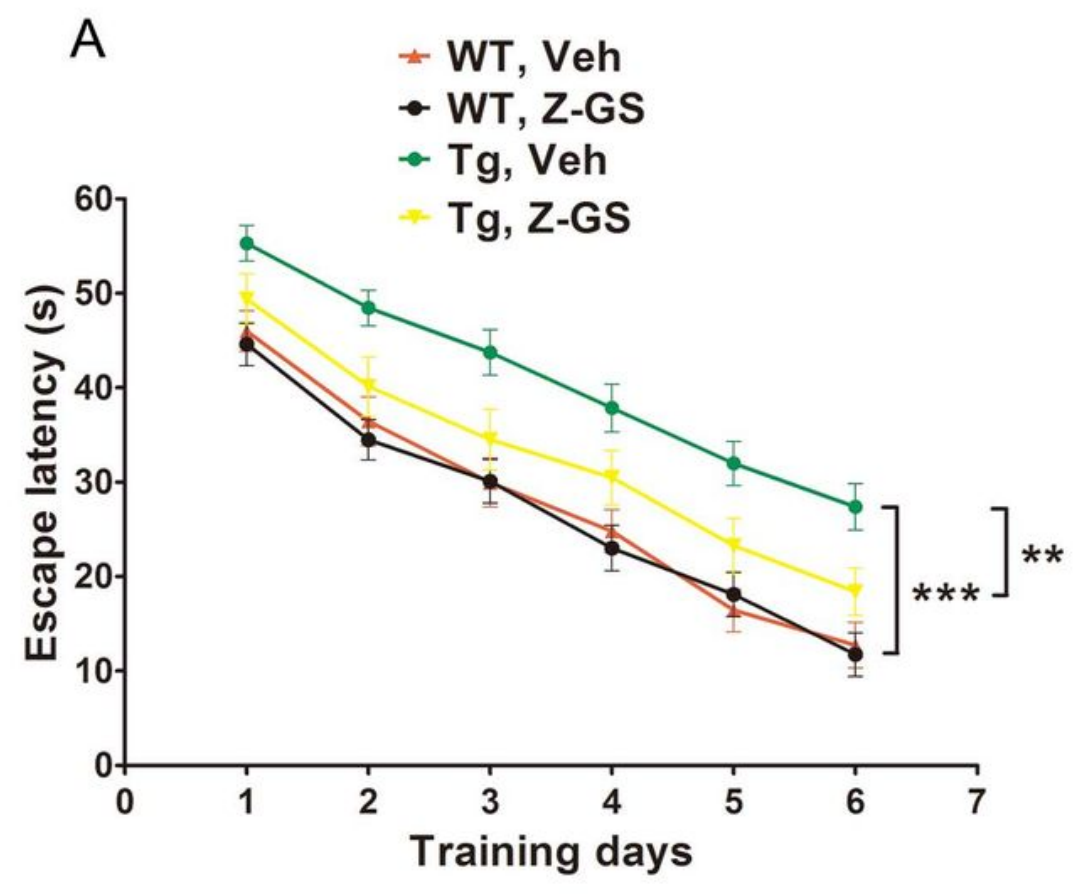

B

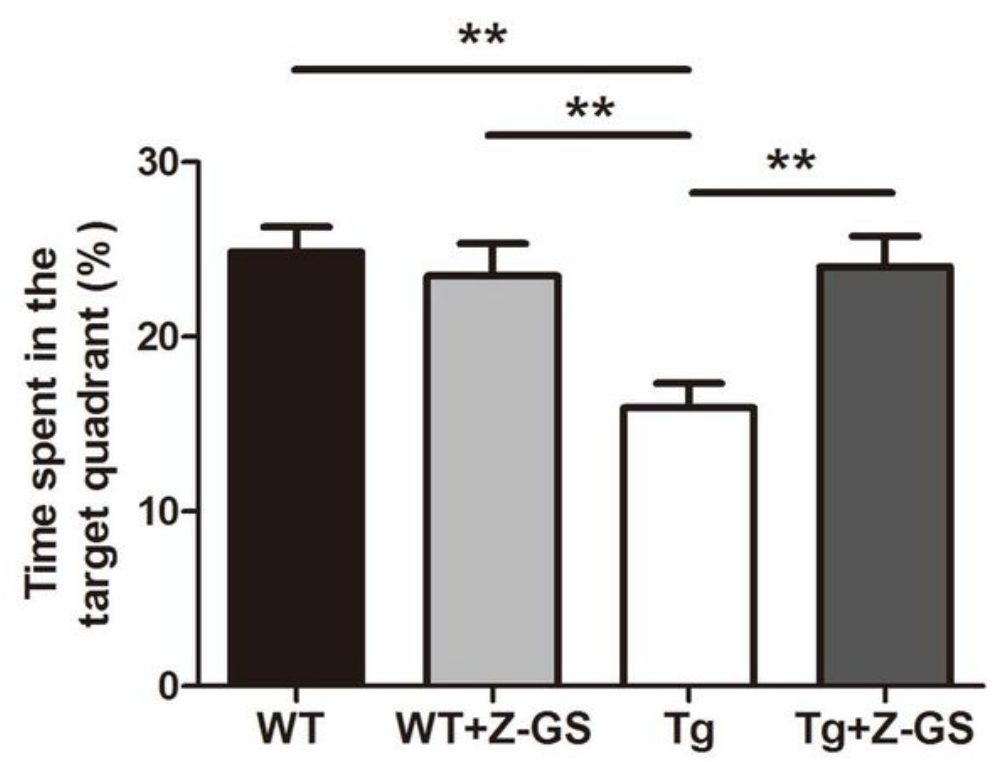

Figure 2

Z-GS treatment improves cognitive deficits in APPswe/PS1dE9 mice. (A) The hidden platform tested for 6 days in the Morris water maze. The escape latency to mount the platform for the 4 groups of mice treated with vehicle or Z-GS as indicated. (B) A probe trial 24 hours after the last hidden platform test in the Morris water maze. The percentages of time spent in the target quadrant for the 4 groups of mice treated 
with vehicle or Z-GS as indicated. Data are presented as mean \pm SEM. $n=7$ mice per group. ${ }^{\star \star} p<0.01$; $\star \star \star p<0.001$.

A

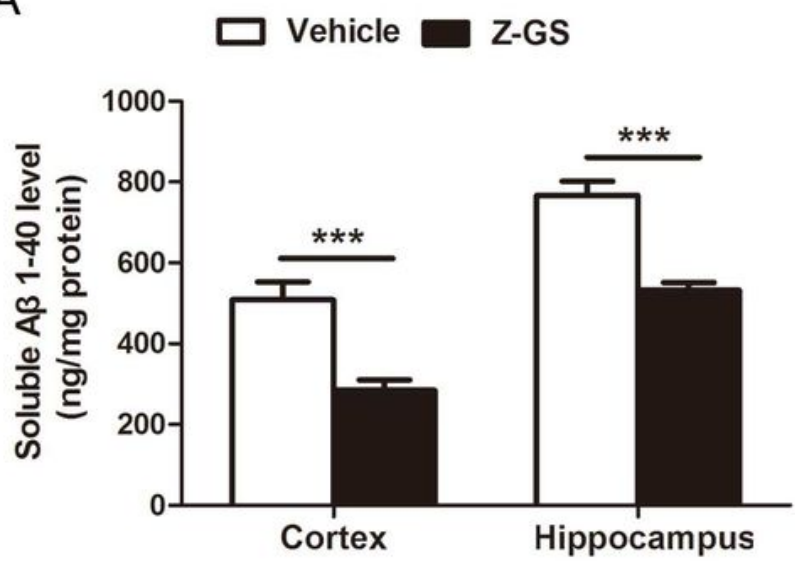

C

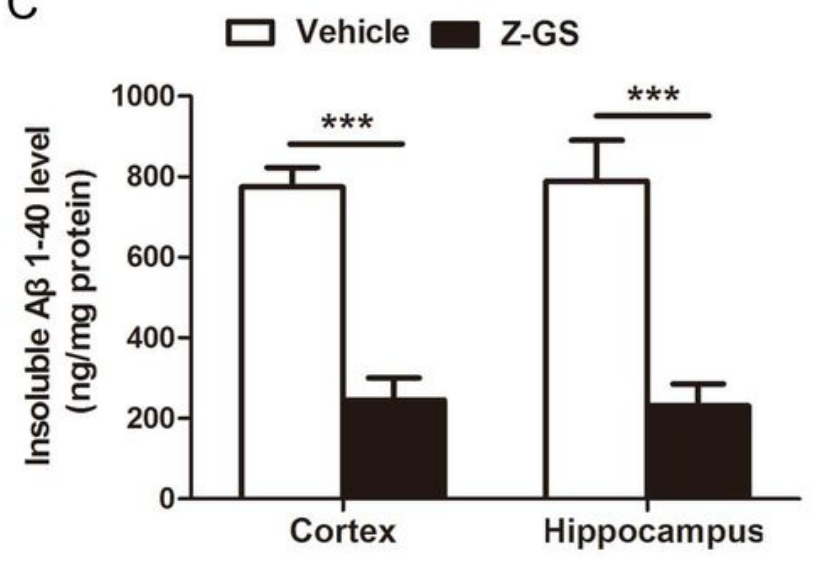

B

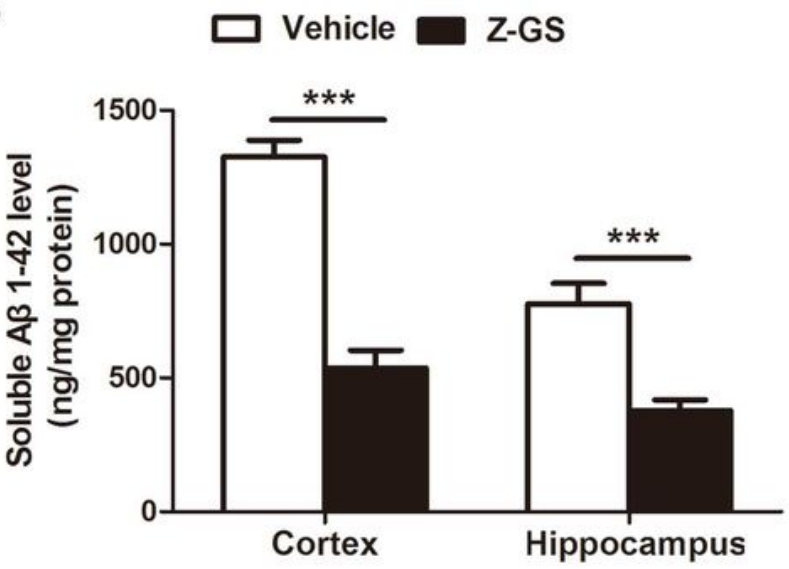

D

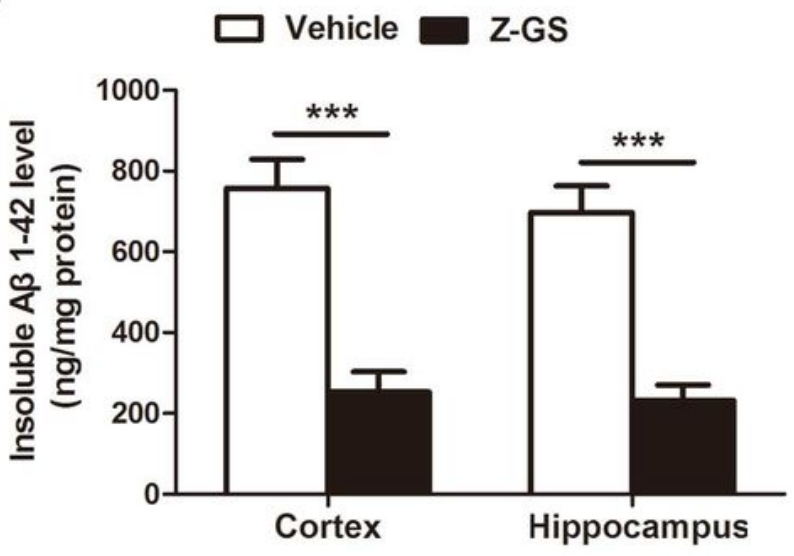

E $\square$ Vehicle

Z-GS

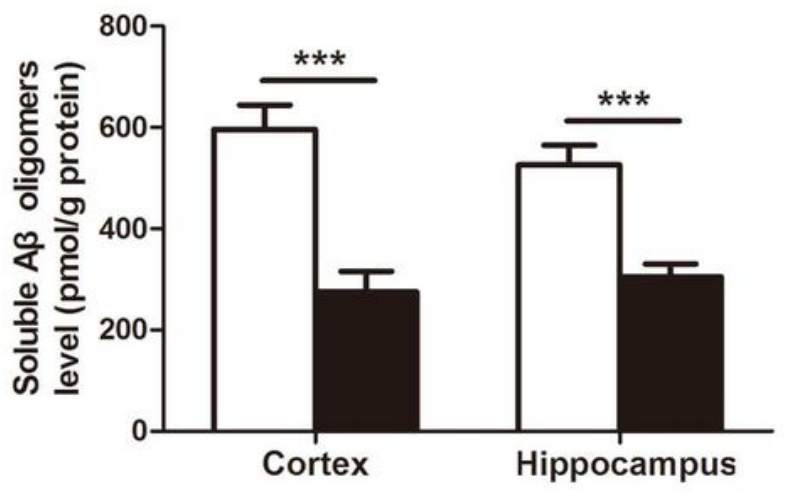

Figure 3

Z-GS treatment decreases brain A $\beta$ levels in APPswe/PS1dE9 mice. The levels of $A \beta$ in the cerebral cortex and hippocampus homogenates from APPswe/PS1dE9 mice treated with vehicle or Z-GS were assayed by commercial ELISA kits. Brain levels of soluble $A \beta 1-40(A)$ and $A \beta 1-42$ (B), insoluble A 1-40 (C) and 
A 1 1-42 (D), and soluble A $\beta$ oligomers (E) in Z-GS-treated APPswe/PS1dE9 mice were significantly lower than in vehicle-treated transgenic controls. Data are presented as mean \pm SEM. $n=7$ mice per group. ${ }^{\star \star \star} p$ $<0.001$.
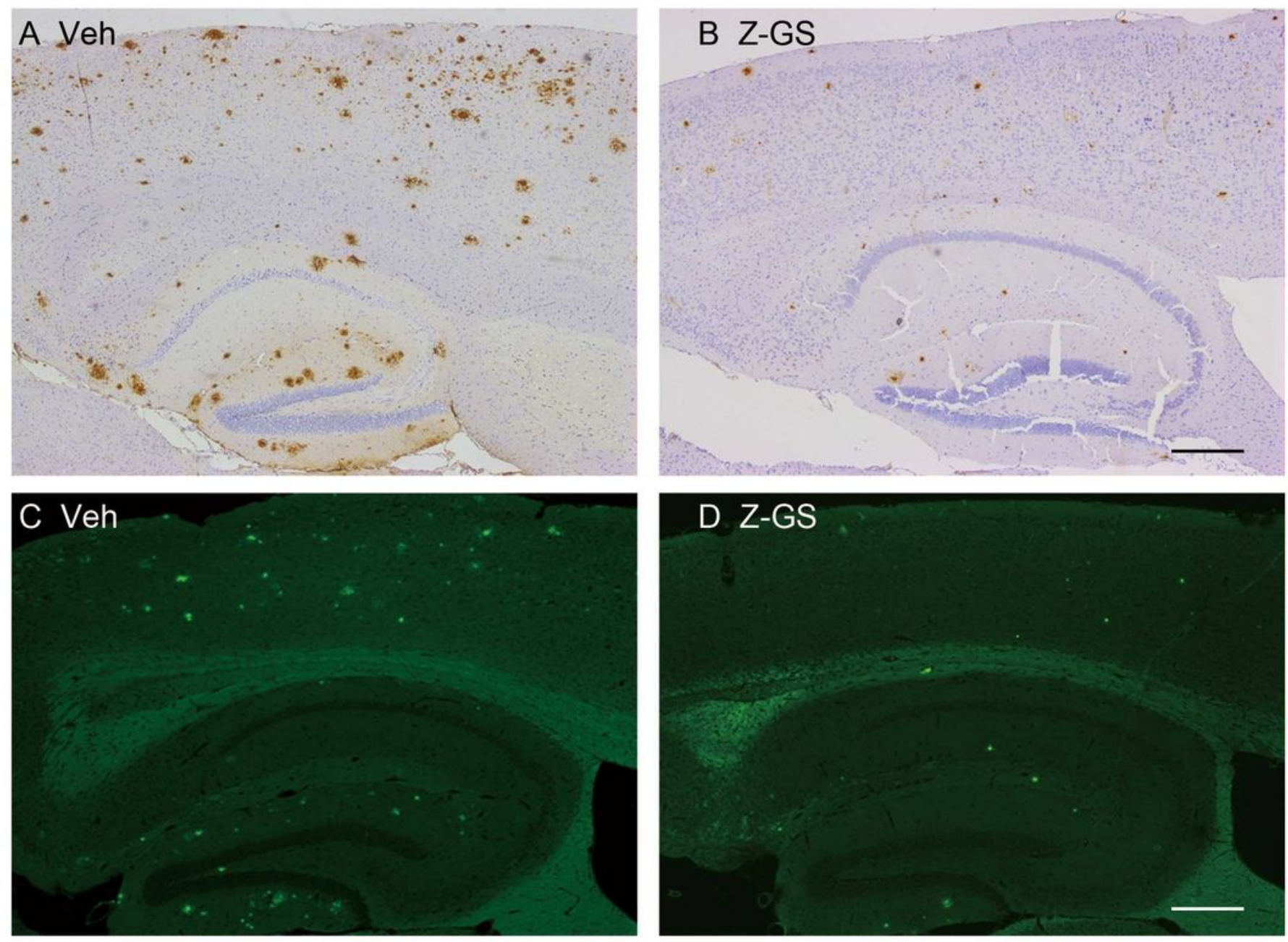

$\mathrm{E}$

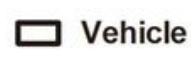

Z-GS

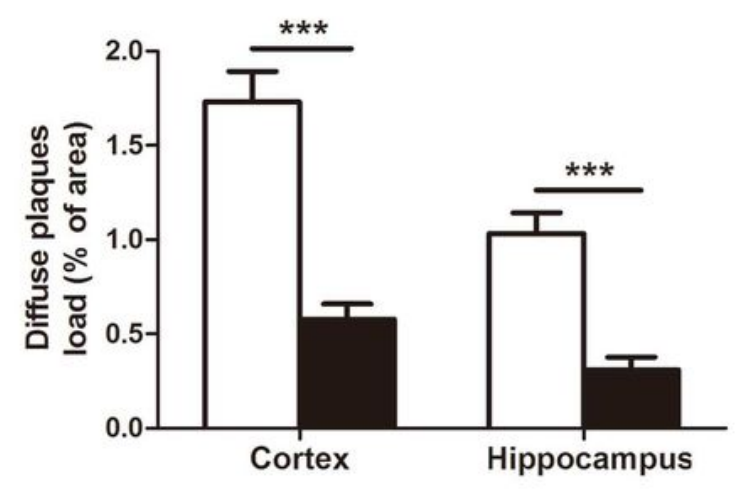

$\mathrm{F}$

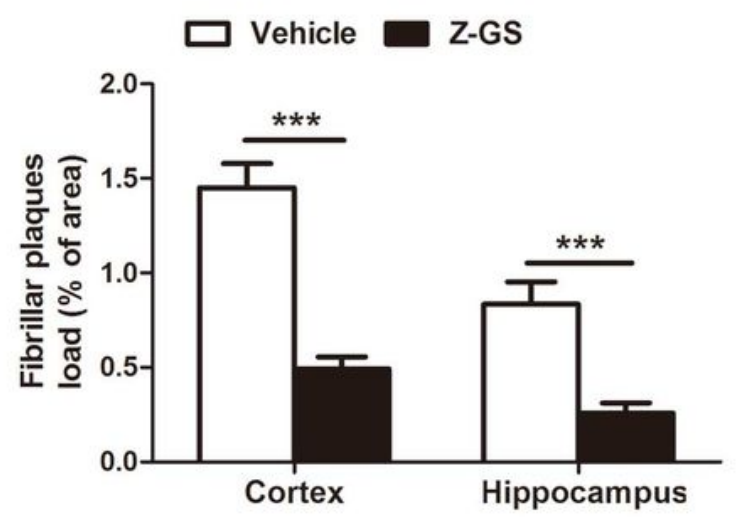

Figure 4

Z-GS treatment reduces amyloid plaque burdens in APPswe/PS1dE9 mice. Representative microphotographs of brain sections from APPswe/PS1dE9 mice treated with vehicle (A and C) or Z-GS (B 
and D) immunostained with Aß1-16 antibody for diffuse plaques (brown) and Thioflavin S for fibrillar plaques (Green). Scale bars, $300 \mu \mathrm{m}$. Quantitative analyses of the percentages of area occupied by diffuse plaques $(E)$ and fibrillar plaques $(F)$ in the brains of APPswe/PS1dE9 mice treated with vehicle or Z-GS were shown. Six sections per mice were analyzed. Data are presented as mean \pm SEM. $n=7$ mice per group. ${ }^{* \star} \mathrm{p}<0.001$.
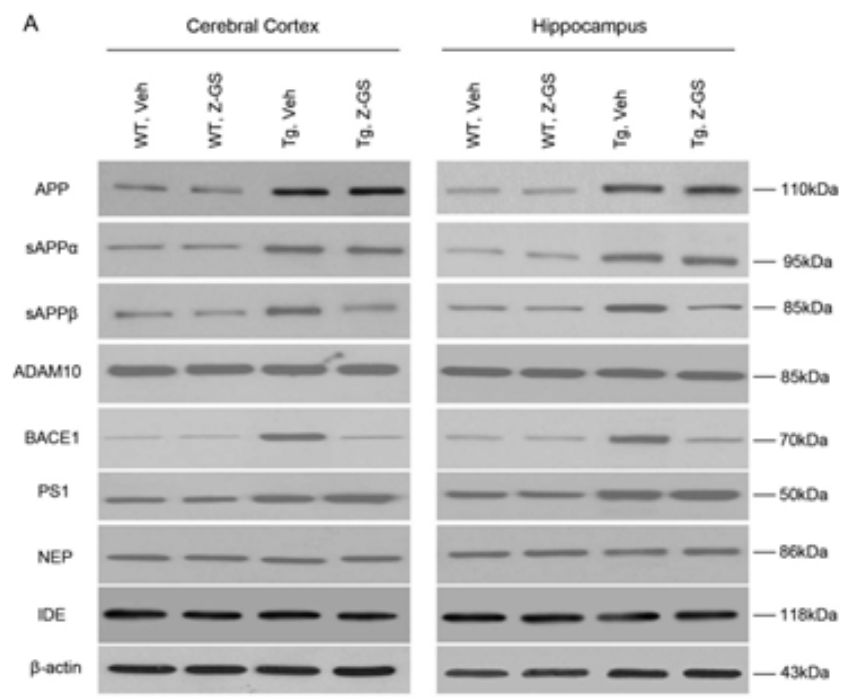

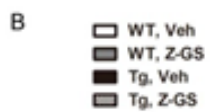

C

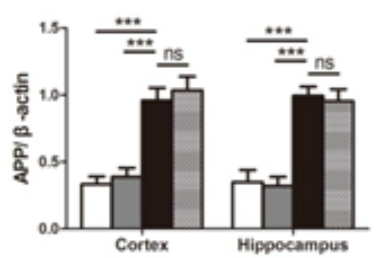

D
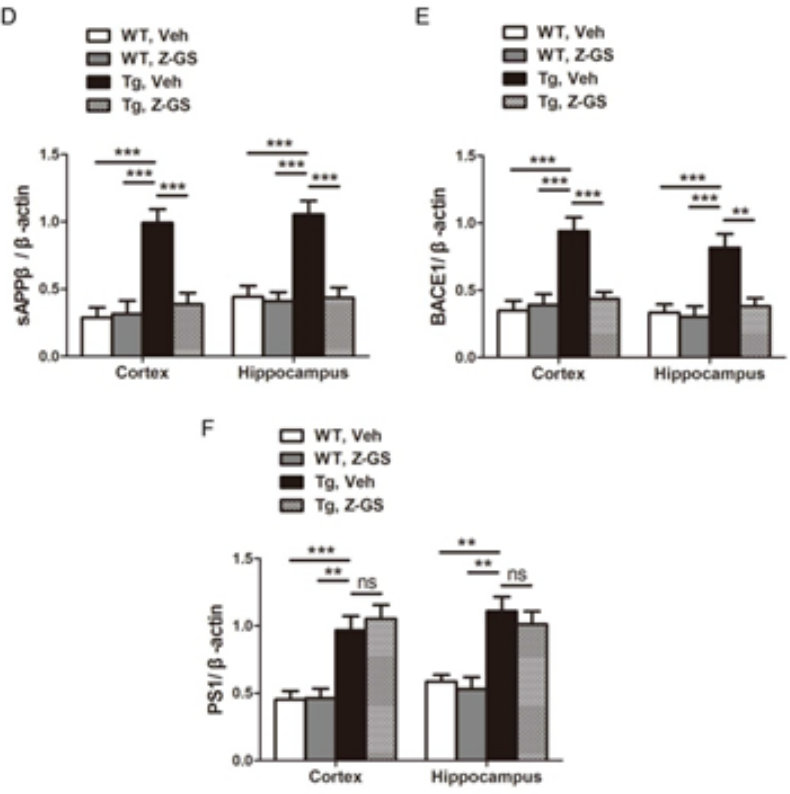

Figure 5 
Z-GS treatment suppresses brain BACE1 expression in APPswe/PS1dE9 mice. (A) Expression of full length APP, sAPPa, SAPPB, ADAM10, BACE1, PS1, NEP, and IDE in the cerebral cortex and hippocampus homogenates from wild-type mice and APPswe/PS1dE9 mice treated with vehicle or Z-GS was examined by western blot. Quantitative analyses of protein band optical densities for full length APP (B), SAPPa (C), SAPP $\beta(D), B A C E 1(E)$, and PS1 (F) expression normalized to $\beta$-actin from each group were shown. Data are presented as mean \pm SEM. $n=7$ mice per group. ${ }^{* \star} p<0.01 ;{ }^{* \star} p<0.001 ; n s$, no significant.
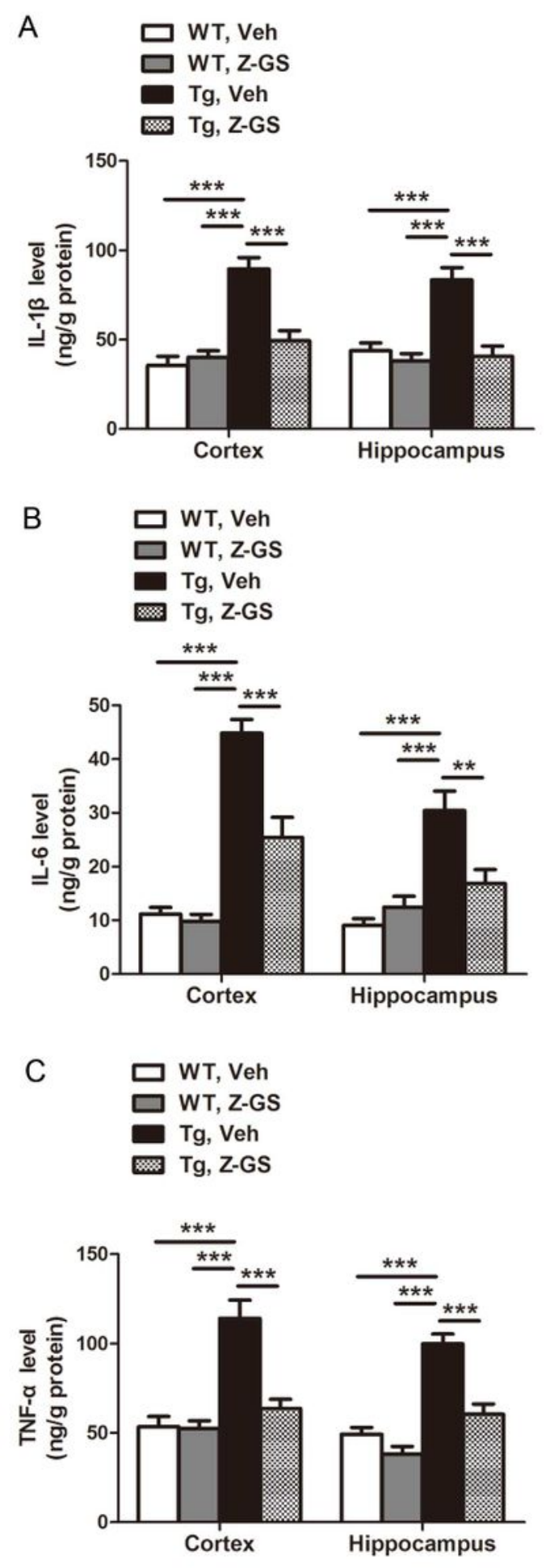

Figure 6 
Z-GS treatment attenuates cerebral inflammation in APPswe/PS1dE9 mice. The levels of IL-1 $\beta$ (A), IL-6 (B), and TNF- $a(C)$ in the brains from wild-type mice and APPswe/PS1dE9 mice treated with vehicle or ZGS were measured by ELISA. Data are presented as mean \pm SEM. $n=7$ mice per group. ${ }^{\star \star} p<0.01 ;{ }^{\star \star \star} p<$ 0.001 .
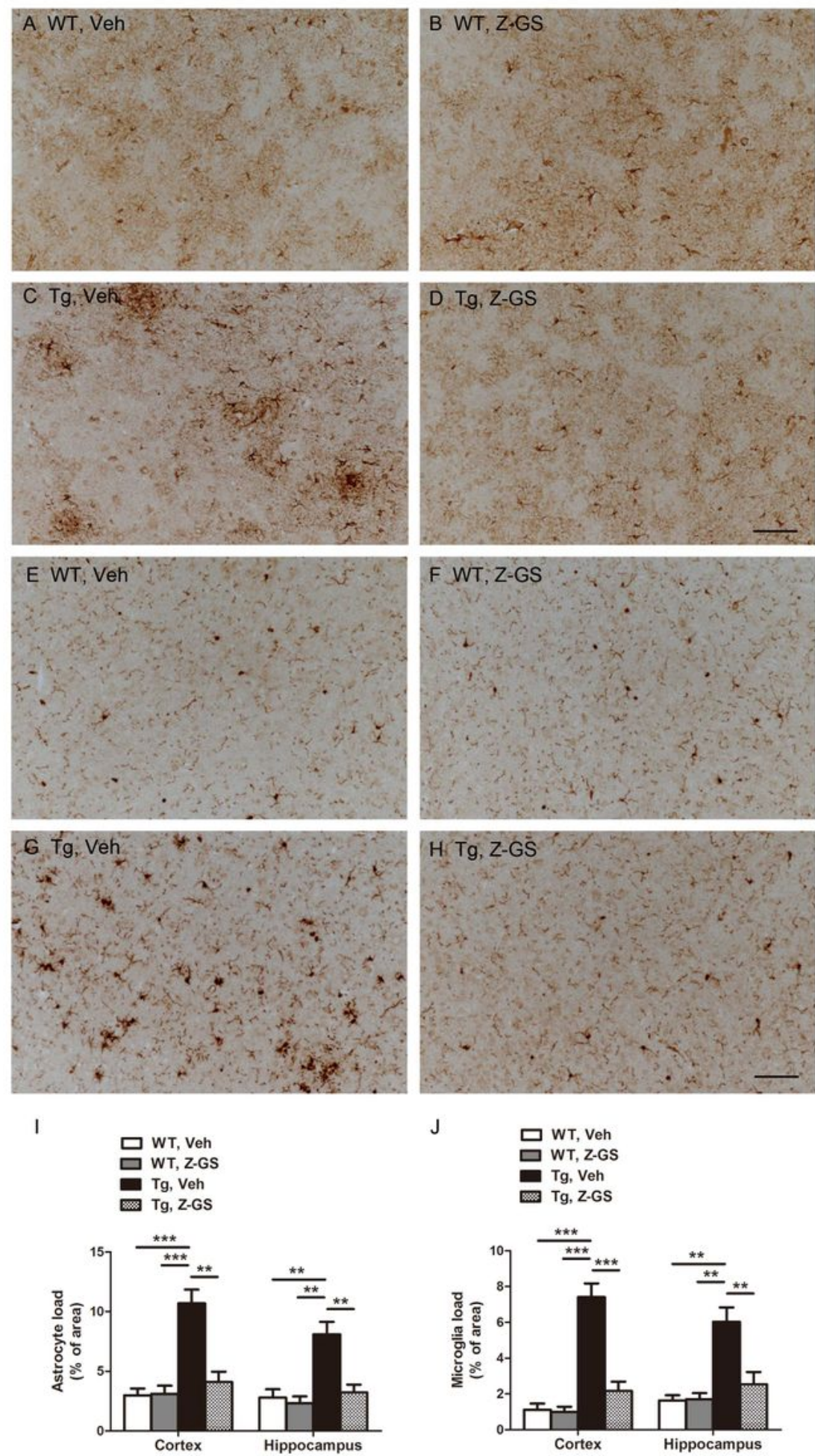

Figure 7 
Z-GS treatment alleviates gliosis in APPswe/PS1dE9 mice. Representative microphotographs of GFAPpositive astrocytes (A-D) and Iba1-positive microglia (E-H) in the frontal cortex of wild-type mice and APPswe/PS1dE9 mice treated with vehicle or Z-GS. Scale bars, $200 \mu \mathrm{m}$. Quantitative analyses of the percentage area occupied by GFAP-positive astrocytes (I) and Iba1-positive microglia $(\mathrm{J})$ in the cerebral cortex and hippocampus. Six sections per mice were analyzed. Data are presented as mean \pm SEM. $n=7$ mice per group. ${ }^{* \star} p<0.01 ; * \star * p<0.001$.
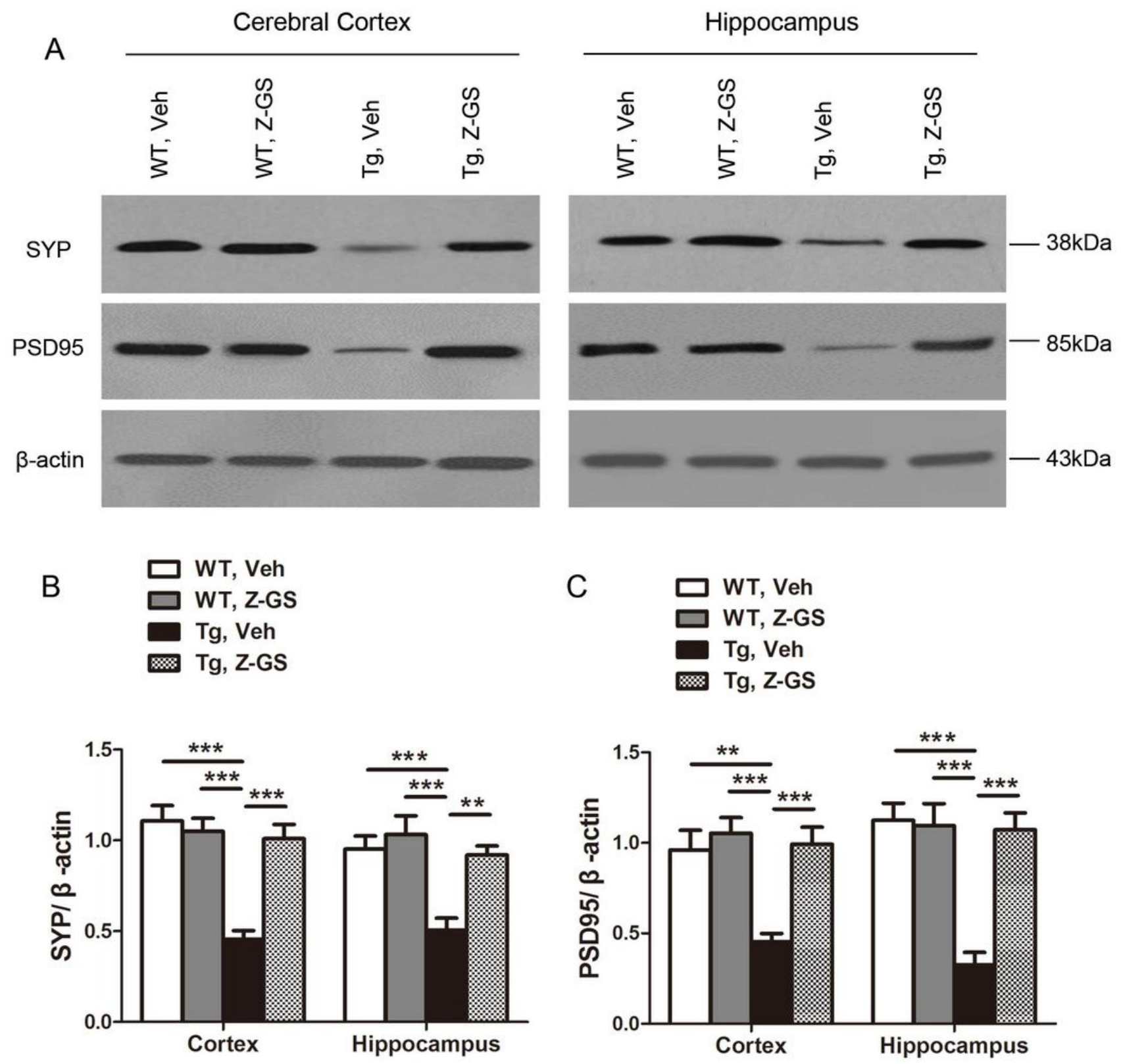

\section{Figure 8}

Z-GS treatment enhances synaptic protein expression in APPswe/PS1dE9 mice. (A) Expression of SYP and PSD95 in the cerebral cortex and hippocampus homogenates from wild-type mice and 
APPswe/PS1dE9 mice treated with vehicle or Z-GS was examined by western blot. Quantitative analyses of protein band optical densities for SYP (B) and PSD95 (C) expression normalized to $\beta$-actin from each group were shown. Data are presented as mean \pm SEM. $n=7$ mice per group. ${ }^{\star \star} p<0.01 ;{ }^{* \star} p<0.001$.

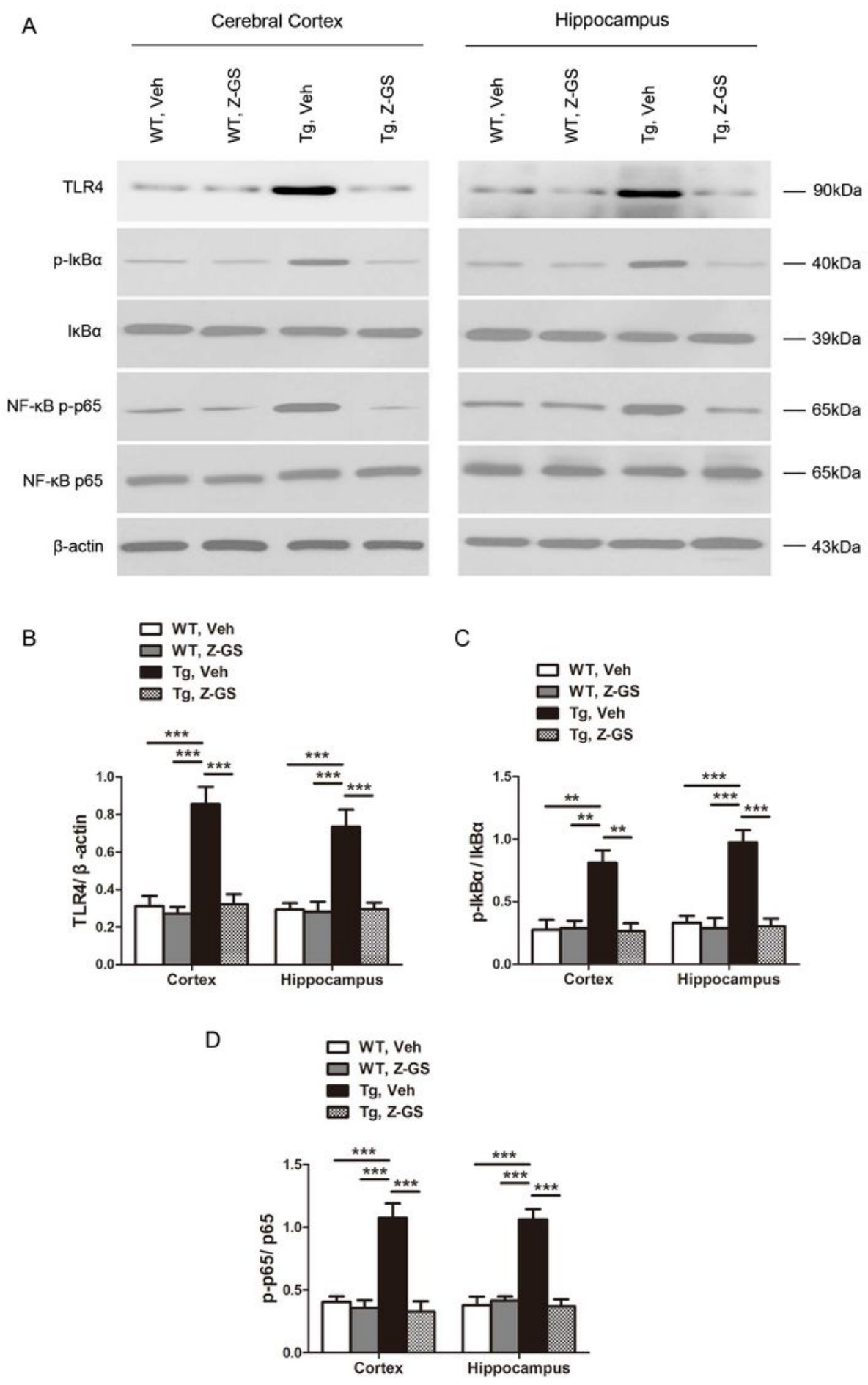

Figure 9

Z-GS treatment inhibits TLR4/NF-kB signaling pathway in APPswe/PS1dE9 mice. (A) Expression of TLR4, p-IkBa, IkBa, p-p65, and p65 in the cerebral cortex and hippocampus homogenates from wild-type 
mice and APPswe/PS1dE9 mice treated with vehicle or Z-GS was examined by western blot. Quantitative analyses of protein band optical densities for TLR4 (B), p-IKBa (C), and p-p65 (D) expression normalized to $\beta$-actin, IKBa, and $\mathrm{p} 65$ respectively were shown. Data are presented as mean \pm SEM. $n=7$ mice per group. ${ }^{* \star} p<0.01 ;{ }^{* \star *} \mathrm{p}<0.001$. 